\title{
THE VALUE OF TECHNETIUM AND GALLIUM SCANNING IN ASSESSING PAIN AFTER TOTAL HIP REPLACEMENT
}

\author{
N. RUSHTON, A. J. COAKLEY, J. TUDOR, E. P. WRAIGHT
}

From Addenbrooke's Hospital, Cambridge

\begin{abstract}
Fifty-one patients with pain after arthroplasty of the hip and 34 control patients were studied using technetium and gallium scintigraphy. Patients with obvious infections were excluded. The method was found to be remarkably accurate in detecting infection or loosening of the prosthesis when compared with conventional techniques and often allowed the diagnosis to be made earlier.
\end{abstract}

Patients with failed prostheses of the hip are presenting for further treatment with increasing frequency. Early diagnosis of the underlying cause of failure is vitally important but differentiation between mechanical and infective loosening is unreliable using conventional methods. Technetium and gallium scanning is an effective method for early differential diagnosis of mechanical loosening and infection.

\section{MATERIALS AND METHODS}

Fifty-one patients presenting at Addenbrooke's Hospital with pain in the hip after total replacement were studied using radioisotope scanning. Patients with obvious infection were excluded, as were patients who had had scans less than 12 months after the arthroplasty in order to minimise the effect of operative trauma on the uptake of the radioisotope.

All 51 patients were scanned approximately four hours after intravenous injection of 15 millicuries (555 megabecquerels) of technetium-99m methylene diphosphonate ( $\left.{ }^{99 \mathrm{~m}} \mathrm{Tc}-\mathrm{MDP}\right)$. An anterior image of the lumbar spine, the whole of the pelvis, both hips and the proximal half of each femur was obtained using an Ohio nuclear camera (Series 410) with a large field of view and a high resolution collimator.

Thirty-six patients also had gallium scans taken approximately two days after an intravenous dose of two to three millicuries (74 to 111 megabecquerels) of gallium-67 citrate. Radionuclide scanning images from the iliac crests to the mid-femur of both legs were obtained using an Elscint rectilinear scanner with a high energy collimator with the energy settings of 70 to 210 kilo-electronvolts.

The scans were interpreted independently by two specialists in nuclear medicine (EPW and AJC) who determined whether there was increased uptake in the femoral or acetabular areas and commented upon the distribution of uptake with reference to the prosthesis. On the basis of these findings, a diagnosis of loosening, with or without infection, was made for both the acetabular and femoral regions. Abnormalities seen on radiographs which could cause confusion in the interpretation of the scans, such as ectopic bone and problems associated with a trochanteric osteotomy, were only revealed after EPW and AJC had reported their findings but before a final diagnosis was made.
In two patients there was a variation in the findings of the reports: these two scans were reported on at the same time as the radiographs and an agreed diagnosis was established.

Radiographs taken up to the time of radionuclide scanning were studied by a consultant radiologist with a special interest in bone and joint radiology (JT); his findings of the acetabular and femoral areas were categorised into normal, loose, loose and infected, or equivocal. Any other abnormalities were recorded and made available to those reporting on the radionuclide scans. Radiographs taken after the scans were used later in the diagnosis of hip pathology, particularly in those cases where no revision operation was performed.

The erythrocyte sedimentation rate (ESR) and a nucleated cell count were available for each patient at the time of scanning. Earlier results of haematology and those obtained up to the time of the revision operation, were used as an aid to diagnosis in all patients. Histological, bacteriological, and clinical examination, both before and at operation, were also used to establish the presence or absence of infection.

The records of the original operation were scrutinised for evidence of infection or haematoma of the wound, or of pyrexia persisting for more than $\mathbf{4 8}$ hours or higher than $\mathbf{3 8}$ degrees Celsius. Other infections, such as urinary tract infections and cholecystitis, which occurred around the time of operation or subsequently, were also recorded. The results of microscopic and bacteriological examination of material obtained from the revision operation, cadavers, or aspiration of the hip were collected.

The medical history of the patient between the original arthroplasty and the onset of pain was extracted carefully to include details of infections and systemic diseases, such as diabetes mellitus. Significant traumatic episodes were also recorded, as were courses of systemic antibiotic therapy, whether given for suspected infection of the hip or for any other reason. Not all the patients in this series had a revision operation so that historical and radiological findings were very important.

\section{RESULTS}

The mean age at the time of scanning was 65 years 8 months (range from 41 years 1 month to 84 years 2 months) and the mean time from operation to the scans was 42.3 months (range 12 to 143 months). The mean

N. Rushton, MA, FRCS, Clinical Lecturer in Orthopaedics, University of Cambridge

J. Tudor, MB, BS, MRCS, LRCP, DMDR, FFR, Consultant Radiologist

E. P. Wraight, PhD, DMRT, Consultant in Nuclear Medicine

Addenbrooke's Hospital, Hills Road, Cambridge CB2 2QQ, England.

A. J. Coakley, MB, ChB, MRCP, MSc, Consultant in Nuclear Medicine

St Thomas' Hospital, Lambeth Palace Road, London SEl 7EH, England.

Requests for reprints should be sent to $\mathrm{Mr}$ N. Rushton.

(C) 1982 British Editorial Society of Bone and Joint Surgery 0301-620X/82/3068-0313\$2.00 
follow-up period after scanning was 19.5 months (range 6 to 36 months). The mean ESR was 25 millimetres in the first hour (range 2 to 103 millimetres). The mean nucleated cell count was $7.4 \times 10^{9}$ cells per litre (range $4.1 \times 10^{9}$ to $13.0 \times 10^{9}$ cells per litre).

The patients were divided into three groups-those whose prostheses were loose but not infected, those who had loosening and infection, and those who had miscellaneous abnormalities.

Loose but not infected. Twenty-five patients had loose prostheses that were not infected; all had technetium scans and 18 had gallium scans. They had a mean age of 64 years 6 months at the time of scanning (range 44 years 10 months to 81 years 6 months). The mean time from the operation to the scans was 51.3 months (range 12 to 143 months) and the mean time of follow-up after scanning was 20.1 months (range 6 to 36 months). The mean ESR was 12.8 millimetres in the first hour (range 2 to 34 millimetres) and the mean nucleated cell count was $7.4 \times 10^{9}$ cells per litre (range $4.8 \times 10^{9}$ to $11.0 \times 10^{9}$ cells per litre). All the patients had received a twocomponent hip replacement: the Charnley arthroplasty was most common but some had McKee-Farrar and Stanmore prostheses. Three patients had metal to metal McKee prostheses.

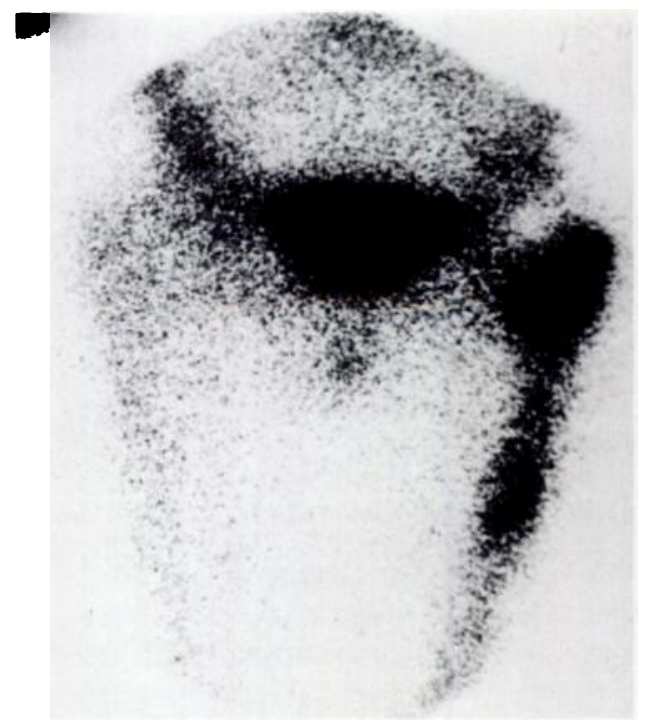

Fig. 1

Technetium scan of a loose femoral stem showing characteristic proximal focal uptake.

Nine patients had acetabular loosening with no evidence of changes around the femoral component. All had considerable radiological evidence of loosening which was confirmed at operation. Two patients had gross destruction of the pelvic bone around the metal McKee acetabular component, and had a slight but significant increase in technetium uptake in the region of the acetabulum which was disproportionate to the amount of loosening and bone loss. This pattern of uptake in the presence of metal to metal joints may be accounted for by reduced osteoblastic activity due to the toxic effect of metal ions, or by loss of bone stock.

Sixteen patients had loosening of the femoral component shown by focal uptake on the technetium scan (Fig. 1). All of the gallium scans were normal. Twelve of the patients had revision operations at which the femoral components were found to be loose. None of these patients had loose acetabular cups at operation. The symptoms of two patients resolved on rest and two patients died before revision could be undertaken. An autopsy specimen from one showed the femoral component to be loose but not infected and that the acetabular component was soundly fixed. Many patients had corroborative radiographic evidence of femoral stem loosening.

Infected. Infected prostheses were diagnosed in 19 patients. All had technetium scans and 13 had gallium scans. Their mean age at the time of scanning was 66 years 11 months (range 51 years 10 months to 81 years). The mean time from operation to the scans was 38.9 months (range 12 to 110 months). The mean follow-up time after scanning was 19.2 months (range 6 to 35 months). The mean ESR was 46 millimetres in the first hour (range 5 to 103 millimetres), and the mean nucleated cell count was $7.8 \times 10^{9}$ cells per litre (range $4.1 \times 10^{9}$ to $13.0 \times 10^{9}$ cells per litre).

All but one of the patients had abnormal technetium scans but all who had gallium scans showed an increased uptake. The patient who had a normal technetium scan had an abnormal gallium scan. On technetium scans, the abnormality most commonly found in the femoral area was focal uptake, characteristic of loosening (Figs 2 and $3)$, but in some grossly infected cases, there was diffuse uptake along the proximal femur (Figs 4 and 5). Technetium scans of infected acetabular areas showed diffuse increase in uptake similar to that seen in association with loose prostheses. Diagnosis of infection was based on having increased uptake on the gallium scan, or diffuse uptake on the technetium scan in the area of the femoral prosthesis or increased uptake in both femoral and acetabular areas.

Eight patients had both areas infected, seven had the femoral area infected, three had the acetabular area infected and one had a localised infection around the greater trochanter. All but one of the patients had infection confirmed by one or more of the conventional methods described previously. Bacteriology sometimes failed to confirm infection even though appropriate techniques were carefully employed; however, many of the patients were taking antibiotics at the time of the operation.

At the time of review the patient whose infection was not confirmed by conventional methods was taking large doses of antibiotics, walking with sticks and had little pain. Bacteriological examination of aspirate from the hip had proved negative but the patient was not well enough to undergo exploration of the hip. 


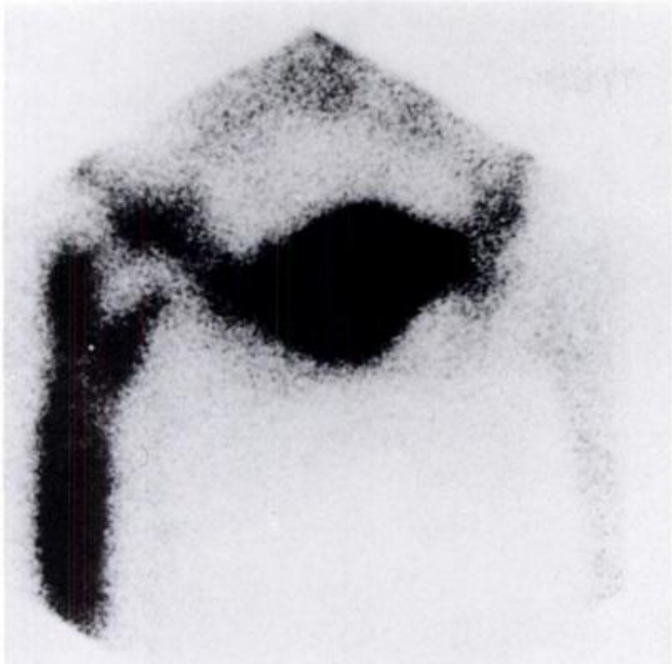

Fig. 2

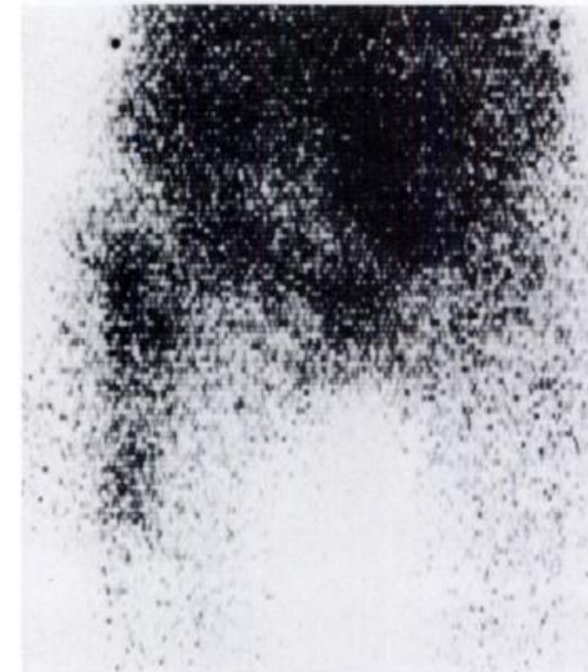

Fig. 3

Technetium and gallium scans of an infected total hip replacement.

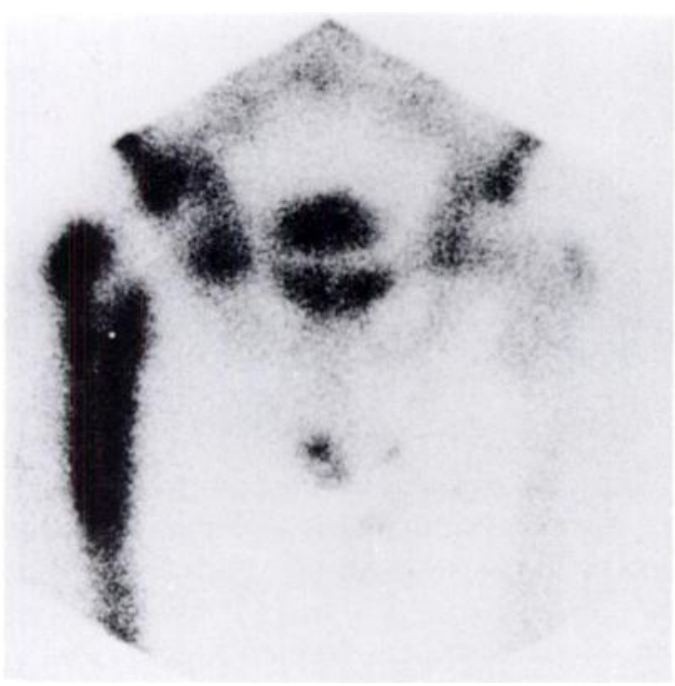

Fig. 4

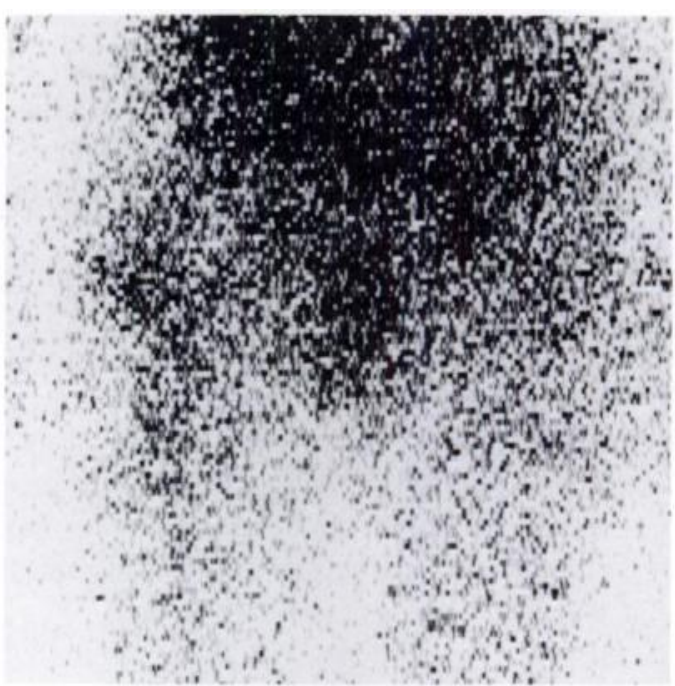

Fig. 5

Technetium and gallium scans of an infected total hip replacement (the technetium scan shows diffuse proximal femoral uptake rather than the figure of eight pattern).

Miscellaneous group. There were seven patients in the miscellaneous group. All had received two-component hip arthroplasties and all had technetium scans. Five also had gallium scans. The mean age at the time of scanning was 66 years 9 months (range 41 years 1 month to 84 years 2 months). The mean time from the most recent operation to the scans was 19.5 months (range 12 to 24 months). The follow-up after scanning varied between 12 and 28 months with a mean of 18.3 months. The mean ESR was nine millimetres in the first hour (range 6 to 16 millimetres) and the mean nucleated cell count was $6.4 \times 10^{9}$ cells per litre (range $4.3 \times 10^{9}$ to $8.6 \times$ $10^{9}$ cells per litre).

This group was separated from the main survey because each had an abnormality demonstrated on the radiograph taken before scanning which was known to produce an increased technetium uptake and which could lead to the wrong diagnosis when interpreting the results of the scan alone. This group consisted of one patient with Paget's disease affecting the pelvis, two patients with ectopic bone formation in the pericapsular area and four patients with non-union of the greater trochanter (Figs 6 and 7). All these abnormalities are seen as localised increases in uptake on the technetium scan. The gallium scan is normal in the presence of these abnormalities which therefore do not interfere with the diagnosis of loosening or infection of a prosthesis.

Control group. A control group of 34 patients with symptom-free total hip replacements was studied. Included in this group were 21 patients from this study with a symptom-free contralateral total hip replacement, eight patients whose painful hips had been scanned but who had been excluded from the study because the problem hip had been operated on less than 12 months before the scan or had been grossly infected, and five patients who had a painful Thompson hemiarthroplasty with a 


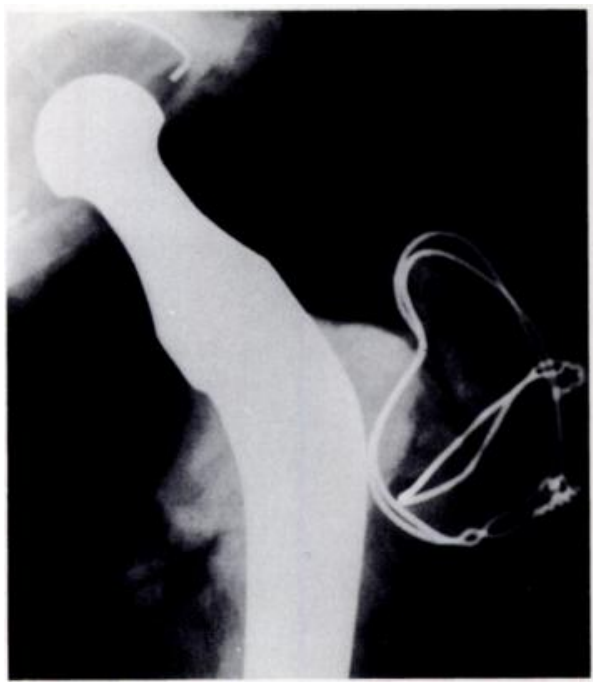

Fig. 6

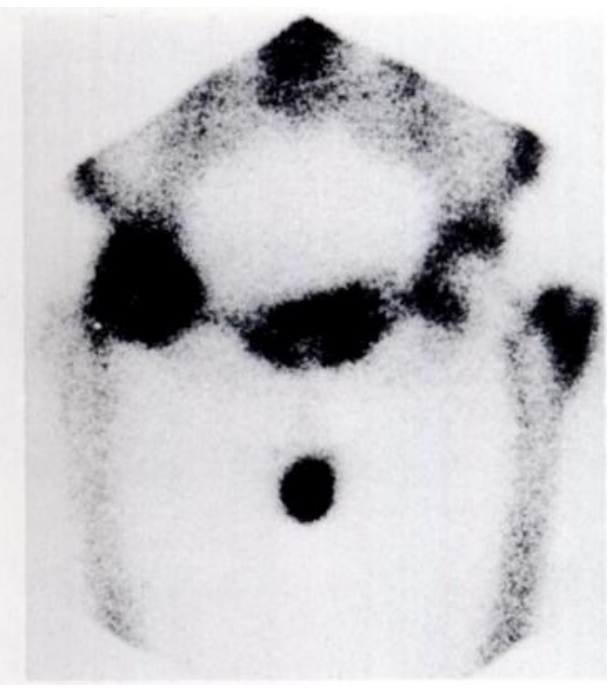

Fig. 7

Radiograph and technetium scan showing non-union of the trochanter

symptom-free contralateral total hip replacement. All patients had technetium scans and 23 had a gallium scan.

The mean age at the time of scanning was 68 years 3 months (range 47 years 2 months to 79 years 10 months) and the mean time from operation to the scans was 38.4 months (range 16 to 143 months). The mean follow-up period after scanning was 18.9 months (range 8 to 78 months). All the gallium scans, and 31 of the technetium scans, were normal. Three of the technetium scans showed the focal uptake characteristic of loosening; these three abnormal technetium scans were not unexpected since it has been shown that painless loosening does occur in hemiarthroplasties (Rushton and Wraight 1980).

\section{DISCUSSION}

This study was undertaken to evaluate the diagnostic accuracy of technetium and gallium scans in differentiating mechanically loose prostheses from infected loose prostheses. The patients included in the series are typical of those seen as orthopaedic outpatients with pain after arthroplasty. We have not included patients in whom there was no diagnostic doubt that the joint was infected, for example those with sinuses, or those with early deep wound infections.

The diagnostic dilemma is clinically important. Revision or exchange arthroplasty is practised increasingly and, if an accurate diagnosis of uninfected loosening can be made before operation, the extent of operative clearance and the meticulous removal of cement can be reduced. The diagnosis of infection can be difficult and has previously relied upon the results of histology and culture of specimens obtained at the revision arthroplasty. Culture of organisms, even when special cultures are made, is not entirely reliable, particularly when systemic antibiotics are being used. Many reports relating to hip infection have been published (Charnley and Eftekhar 1969; Buchholz and Gartmann 1972; Artz et al. 1975; Burton and Schurman 1975; Hunter and Dandy 1977; Andrews et al. 1981). Definitions of deep infection have varied throughout these papers. The negative results of bacteriology in hips which have the histological, clinical and macroscopic appearance of infection has resulted in the hips being described as aseptic or as chemically inflamed.

Lindberg (1979) has shown that 10 per cent of biopsies from untreated hips can provide positive cultures of organisms similar to those commonly found in infected hip arthroplasties. An unexpected positive culture may be explained in this way. Patients had often started antibiotic treatment before scanning and continued to take antibiotics until long after operation. All our infected group were taking antibiotics at the time of the gallium scan which may have changed the character of the uptake but, as is obvious from the success rate, did not interfere with the diagnostic efficiency of the scan.

Haematological testing was found to be of limited value. A markedly elevated ESR or nucleated cell count suggested infection. The mean results within the groups confirms this trend, but individual results could not be relied upon to distinguish between infection and loosening. Other disease processes could have led to elevation of the ESR and the nucleated cell count, particularly in the older patients in the series.

Conventional radiography, particularly the study of sequential films, was of some value (Salvati et al. 1976; Bergström, Lidgren and Lindberg 1974; Gelman et al. 1978). Radiography could demonstrate loosening only when it was relatively advanced and was not as sensitive a test as technetium scanning. Differentiation between mechanical loosening and infective loosening was at best speculative, and in our series unreliable. Radiography could detect infection but not before substantial loss of 
bone and spread of infection had occurred which is when diagnosis is required. Radiography was valuable in the diagnosis of the known miscellaneous conditions which produced increase in uptake of technetium which otherwise could lead to the wrong diagnosis.

Technetium scanning alone is valuable in the diagnosis of loosening, whether mechanical or infective. Early workers using strontium and technetium were not able to differentiate between mechanical loosening and infection and most equated increased uptake with infection (Feith et al. 1976; Williams, Tregonning and Hurley 1977; McInerney and Hyde 1978; Ostrowski, Wilkinson and Browett 1978; Weiss et al. 1979). We have demonstrated previously that focal uptake around the femoral prosthesis (Fig. 1) is indicative of loosening (Rushton and Wraight 1980); this has been confirmed by other workers (Weiss et al. 1979). Early infection is indistinguishable from loosening on technetium scans (compare Figures 1 and 2). In more advanced infection, focal distribution is replaced by uniform increase in uptake in the proximal femur (Fig. 4) which presumably represents osteomyelitis in this area. If both the femoral and acetabular components show increased uptake on the technetium scan, it is more likely that the joint is infected.

Gallium is taken up by a number of tissues (Larson 1978; Staab and McCartney 1978; Tsan et al. 1978; Bekerman et al. 1980) and can be used to detect infected tissues (Norris et al. 1978) and tumours. It has also been used successfully to diagnose and follow-up osteomyelitis (Deysine et al. 1975a,b; Kolyvas et al. 1978). The increased uptake of gallium in the area of infection is due to its affinity to polymorphonuclear leucocytes, which increase in number at the site of infection, rather than to any of the other pathological conditions for which it has an affinity. The affinity of gallium to polymorphonuclear leucocytes is probably a result of its binding to lactoferrin (Weiner, Hoffer and Thakur 1981).

One patient had a normal technetium scan but a positive gallium scan and infection was confirmed at operation. This is an unexpected and so far unexplained finding which has not been repeated in subsequent patients who are not included in this report. We feel that gallium scanning is indicated in the presence of a positive technetium scan. A positive gallium scan was entirely accurate in the diagnosis of infection in our series, the one unproven patient having been diagnosed on technetium scan alone.

A long follow-up without antibiotics was required to establish that there were no false negative gallium scans, particularly in those patients whose hips were not explored surgically. Patients with mild infections, who were taking antibiotics and resting their hips, were difficult to assess by any method.

Similar findings have been reported elsewhere (Reing, Richin and Kenmore 1979; Rosenthall et al. 1979; Horoszowski et al. 1980; McCall, Williams and Parke 1980; Rushton et al. 1980). We feel that the combined use of technetium and gallium scanning is an accurate and reliable method of demonstrating infection surrounding arthroplasties of the hip. Technetium scanning alone demonstrates loosening and in some cases can give a good indication of infection but it is not as reliable in differentiating between mechanical loosening and infected loosening as is the combination of a technetium and a gallium scan.

We would like to acknowledge the help of Mr A. H. G. Murley, Mr. P. M. Scott, Mr B. F. Meggit, Mr D. J. Dandy and Mr M. H. Matthewson for permission to study their patients. We thank the staff of the Nuclear Medicine and Medical Photography Departments and the secretarial help of Miss Sally Whitehouse and Mrs Sarah Jarvis.

\section{REFERENCES}

Andrews HJ, Arden GP, Hart GM, Owen JW. Deep infection after total hip replacement. J Bone Joint Surg [Br] 1981;63-B:53-7.

Artz TD, Macys J, Salvati EA, Jacobs B, Wilson PD Jr. Hematogenous infection of total hip replacements. J Bone Joint Surg [Am] 1975; 57-A: 1024.

Bekerman C, Hofier PB, Bitran JD, Gupta RG. Gallium-67 citrate imaging studies of the lung. Semin Nucl Med 1980:10:286-301

Bergström B, Lidgren L, Lindberg L. Radiographic abnormalities caused by postoperative infection following total hip arthroplasty. Clin Orthop 1974:99:95-102.

Buchholz HW, Gartmann H-D. Infektionsprophylaxe und operative Behandlung der schleichenden tiefen infektion bei der totalen Endoprothese. Chirurg 1972:43:446-53.

Burton DS, Schurman DJ. Hematogenous infection in bilateral total hip arthroplasty. J Bone Joint Surg [ Am] 1975;57-A : 1004-5.

Charnley J, Eftekhar N. Postoperative infection in total prosthetic replacement arthroplasty of the hip-joint with special reference to the bacterial content of the air of the operating room. Br J Surg 1969:56:641-9.

Deysine M, Rafkin H, Russell R, Teicher I, Aufses AH Jr. The detection of acute experimental osteomyelitis with ${ }^{67} \mathrm{Ga}$ citrate scannings. Surg Gynecol Obstet 1975a:141:40-2.

Deysine M, Rafkin H, Teicher I, Silver L, Robinson R, Manly J, Aufses AH Jr. Diagnosis of chronic and postoperative osteomyelitis with gallium67 citrate scans. Am J Surg 1975b:129:632-5.

Feith R, Slooff TJJH, Kazem I, van Rens TJG. Strontium ${ }^{87 \mathrm{~m}}$ Sr bone scanning for the evaluation of total hip replacement. J Bone Joint Surg [Br] 1976:58-B:79-83.

Gelman MI, Coleman RE, Stevens PM, Davey BW. Radiography, radionuclide imaging, and arthrography in the evaluation of total hip and knee replacement. Radiology 1978;128:677-82.

Hunter G, Dandy D. The natural history of the patient with an infected total hip replacement. J Bone Joint Surg [Br] 1977;59-B:293-7.

Horoszowski H, Ganel A, Kamhin M, Zaltzman S, Farine I. Sequential use of technetium-99m MDP and gallium-67 citrate imaging in the evaluation of painful total hip replacement. Br J Radiol 1980;53:1169-73.

Kolyvas E, Rosenthall L, Ahronheim GA, Lisbona R, Marks MI. Serial ${ }^{67}$ Ga-citrate imaging during treatment of acute osteomyelitis in childhood. Clin Nucl Med 1978:3:461-6.

Larson SM. Mechanisms of localization of gallium-67 in tumors. Semin Nucl Med 1978:8:193-203. 
Lindberg L. Revision arthroplasty in Scandinavia. In: Elson RA, Caldwell ADS eds. Revision arthroplasty. Proceedings of a symposium held at Sheffield University. Oxford: Medical Education Services Limited, 1979: 17-24.

McCall IW, Williams F, Parke WM. Gallium-67 scanning in the painful hip replacement. Nucl Med Commun 1980;1:184.

McInerney DP, Hyde ID. Technetium ${ }^{99} \mathrm{Tc}^{\mathrm{m}}$ pyrophosphate scanning in the assessment of the painful hip prosthesis. Clin Radiol 1978;29:513-7. Norris S, Ehrlich MG, Keim DE, Guiterman H, McKusick KA. Early diagnosis of disc-space infection using gallium-67. J Nucl Med 1978:19:3846.

Ostrowski ST, Wilkinson JM, Browett JP. Radioisotope scanning after total hip replacement. In : Radioaktive isotope in clinik und forschung. (13th international symposium on radioactive isotopes in clinical medicine) Vienna: Egerman, 1978: 453-9.

Reing CM, Richin PF, Kenmore PI. Differential bone-scanning in the evaluation of painful total joint replacement. J Bone Joint Surg [Am] $1979 ; 61-A: 933-6$.

Rosenthall L, Lisbona R, Hernandez M, Hadjipavlou A. ${ }^{99 \mathrm{~m}} \mathrm{Tc}-\mathrm{PP}$ and ${ }^{67} \mathrm{Ga}$ imaging following insertion of orthopedic devices. Radiology 1979:133:717-21.

Rushton N, Coakley AJ, Tudor J, Wraight EP. The value of bone and gallium scanning in assessment of the painful hip replacement. Nucl Med Commun 1980;1:183

Rushton N, Wraight EP. Technetium 99m methylene diphosphonate scanning in Thompson hemiarthroplasties. Br J Radiol 1980:53:781-3.

Salvati EA, Chuem IV, Aglietti P, Wilson PD Jr. Radiology of total hip replacements. Clin Orthop 1976;121:74-82.

Staab EV, McCartney WH. Role of gallium-67 in inflammatory disease. Semin Nucl Med 1978;8:219-34

Tsan M-F, Chen WY, Scheffel G, Wagner HN Jr. Studies on gallium accumulation in inflammatory lesions. I: Gallium uptake by human polymorphonuclear leukocytes. $J$ Nucl Med 1978;19:36-43.

Weiner R, Hofier PB, Thakur ML. Lactoferrin: its role as a Ga-67-binding protein in polymorphonuclear leukocytes. J Nucl Med 1981;22:32-7.

Weiss PE, Mall JC, Hoffer PB, Murray WR, Rodrigo JJ, Genant HK. 99m Tc-methylene diphosphonate bone imaging in the evaluation of total hip prostheses. Radiology 1979; 133:727-30.

Williams ED, Tregonning RJ, Hurley PJ. ${ }^{99} \mathrm{Tc}^{\mathrm{m}}$-diphosphate scanning as an aid to diagnosis of infection in total hip joint replacements. $B r J$ Radiol 1977:50:562-6. 